\section{The gut microbiome and cardiovascular risk: current perspective and gaps of knowledge}
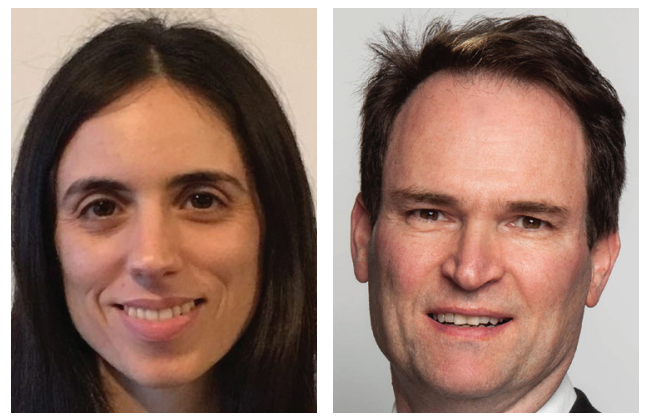

"This editorial aims at providing a brief overview of recent evidence of gut microbiota-host metabolic interactions with a focus on cardiovascular disease and cardiovascular risk factors.”

Yeela Talmor-Barkan ${ }^{* 1,2} \&$ Ran Kornowski ${ }^{1,2}$

First draft submitted: 6 March 2017; Accepted for publication: 10 March 2017; Published online: 31 May 2017

Heart disease is the leading cause of death for both men and women, taking the lives of approximately 610,000 people in the USA every year [1]. Coronary heart disease is the most common type of heart disease, killing over 370,000 people annually [1], despite considerable advances in the treatment. In the quest to improve outcomes in patients with cardiovascular disease (CVD), research has focused on novel modifiable risk factors, with a special attention to environmental factors. Recent findings have implicated the gut microbiota as a contributor of metabolic diseases through the modulation of host metabolism and highlight the participation of gut microbes in the pathogenesis of CVD [2].

This editorial aims at providing a brief overview of recent evidence of gut microbiota-host metabolic interactions with a focus on CVD and cardiovascular risk factors. Furthermore, this piece will emphasize prospective applications such as predication algorithms based on microbiome signature and treatments targeting gut microbiota for means of primary and secondary prevention.

The link between gut microbiome \& human metabolic processes

The human gastrointestinal system is populated with a variety of symbiotic microorganisms, namely microbiota. Its total weight is approximately $2 \mathrm{~kg}$, containing trillions of microorganisms [3]. The microbiome is the total genetic (metagenomic) data of the microbiota. Recent studies have estimated that the microbiota genome contains 100-fold more genes than the host genome [4]. In recent years, the development of efficient methods for genome sequencing and bioinformatics has enabled fast and accurate quantification and qualification of

\section{KEYWORDS}

- coronary artery disease $\bullet$ ischemic heart disease $\bullet$ microbiome

- microbiota • personalized medicine

- TMAO • vascular disease 


\author{
"Gut microbiota is an \\ important environmental \\ factor involved in the \\ regulation of body weight \\ and energy homeostasis."
}

the microbiome and made microbiome analysis a leading method of microbiota research [5]. The human gut microbiota interacts extensively with the host through metabolic exchange and co-metabolism of substrates; thereby contributing to a variety of metabolic and immunologic mechanism in the human body [6].

The integration of metagenomic data with the analysis of metabolic processes provides deeper understanding of the metabolic capabilities of the metagenome. Since most of the traditional CVD risk factors, such as obesity, dyslipidemia and insulin resistance, have a metabolic origin, this approach is suitable for delineating the link between the gut metagenome and cardiovascular risk factors and disease. A prime example is the pro-atherogenic and prothrombotic plasma metabolite trimethylamine $\mathrm{N}$-oxide (TMAO). TMAO is shown to be formed through a metaorganismal pathway involving nutrient precursors abundant in a Western diet (choline, phosphatidylcholine and L-carnitine) and the sequential action of both gut microbiota, initially forming trimethylamine, followed by host hepatic flavin monooxygenase-dependent conversion into TMAO [7].

\section{The gut microbiome, CVD \& risk factors}

Knowing the significant contribution of the environmental component to CVD and the established link between gut microbiome and human metabolic processes, CVD has become a major field of interest in microbiota research. There have been several findings that connect the gut microbiota to CVD pathophysiology.

First, microbiota is associated with traditional CVD risk factors - Type 2 diabetes mellitus (T2DM) and metabolic syndrome (namely obesity and insulin resistance). Gut microbiota is an important environmental factor involved in the regulation of body weight and energy homeostasis. By using an obese mouse model, Turnbaugh et al. demonstrated that the microbiome associated with obese mice was capable of extracting more calorific value from food compared with lean animals, and this obese phenotype was transferable by the transplantation of feces into germ-free mice [8]. Another model using germ-free mice showed that in contrast to mice with a gut microbiota, germ-free mice are protected against obesity that develops after consuming a Western-style, high-fat and sugar-rich diet [9]. Several clinical and animal studies have demonstrated that the gut microbiota and their imbalance state, dysbiosis, are associated with T2DM. A small double-blinded randomized controlled trial in insulin-resistant males with metabolic syndrome assigned 18 subjects to groups that were given small intestinal infusions of allogenic or autologous microbiota from lean donors (nine in each group). Six weeks after infusion of microbiota from lean donors, insulin sensitivity of recipients increased. This change was accompanied by a significantly increased intestinal microbial diversity, along with a distinct increase in levels of butyrate-producing bacteria, such as Roseburia and Eubacterium halii [10].

Second, microbiota may directly contribute to the development of atherosclerosis. One hypothesis is that gut microbiota endotoxins may translocate into the bloodstream and elicit the inflammatory cascade that eventually promotes atherosclerosis. Interestingly, oral bacteria are associated with increased CVD, while the same bacteria that are associated with oral cavities have been found in atherosclerotic plaques [11]. In patients with symptomatic atherosclerosis, there is a unique microbiome pattern that may have pro-inflammatory characteristics [12]. A unique microbiome signature was also demonstrated among patients with high cardiovascular risk profile [13]. As for microbiota analysis of patients with coronary artery disease (CAD), Emoto et al. demonstrated a characteristic change of their gut microbiota compared with healthy volunteers. The order Lactobacillales was increased, whereas the phylum Bacteroidetes was decreased in the CAD group [2]. Nevertheless, except for the latter study, data regarding microbiome analysis of patients with established diagnosis of CAD (including acute coronary syndrome) is lacking.

Third, there is evidence of a causal link between microbiome and CVD, by the discovery of plasma metabolites formed through a metaorganismal pathway involving dietary intake, gut microbiota and liver metabolism, such as TMAO [14]. Numerous studies reveal an association between systemic TMAO levels and cardiovascular risk in both humans and animals [7]. TMAO interacts with platelets, inducing platelet hyper-reactivity and thereby increasing thrombosis potential. Both in vivo studies in mice and in vitro studies in vasculature demonstrated that TMAO induces vascular inflammation by induction of pro-inflammatory cytokines expression, leukocyte recruitment and increase in adhesion molecules [15]. Using a mouse model, researchers managed to prevent atherosclerosis by 
decreasing plasma TMAO levels [16]. Recently, the group of Stanley Hazen showed that plasma TMAO levels among patients presenting with acute coronary syndrome may predict both near and long-term adverse cardiovascular events [17].

In addition to atherosclerosis, patients with heart failure (HF) have significantly higher levels of TMAO compared with age and sex-matched non-HF subjects and elevated plasma TMAO portends poorer long-term survival, regardless of underlying etiology [18].

\section{Future perspective}

The individual composition of gut microbiota is affected by host diet, lifestyle, hygiene and host genetics, making the microbiome a dynamic environmental factor and a therapeutic target for multifactorial diseases including T2DM, metabolic syndrome and even CVD.

There are many ways to alter the gut microbiota including probiotics (nonpathogenic organisms beneficial to the host), prebiotics (chemicals that induce growth or activity of commensal organisms) and fecal microbiota transplantation (FMT). The beneficial effects of probiotics have been demonstrated in several studies; nevertheless they did not show significant alteration in fecal microbiota composition [19]. FMT on the other hand, causes significant changes in fecal microbiota composition. The first clinical use of FMT was for the treatment of patients with pseudomembranous colitis, an unremitting infection with Clostridium difficile [19]. Recent evidence from animal models suggests FMT as a therapeutic intervention against metabolic disorders [20]. However, as a therapeutic intervention against metabolic disorders in humans, the data regarding FMT are limited, and to date only one small study was published on the subject, as mentioned earlier [10]. Additional long-term clinical trials are necessary to validate the effects of FMT in patients with metabolic diseases.

Regarding predication, can our gut microbiome predict CVD? Karlsson et al. characterized the fecal metagenome of 145 European women with normal, impaired or diabetic glucose control. They observed compositional and functional alterations in the metagenomes of women with T2DM and accordingly developed a mathematical model based on metagenomic profiles that identified T2DM with high accuracy. They applied this model to women with impaired glucose tolerance and showed that it can identify women who have a diabetes-like metabolism and are at high risk of developing type T2DM [21].

Those diagnostic and therapeutic concepts are defiantly gaining traction, however, further work is needed before our understanding of altered intestinal microbiota can be used in these settings.

\section{Conclusion}

In recent years, increasing evidence has indicated that host-gut microbiome interactions play a significant role in health and diseases of the host, with obesity, T2DM and metabolic syndrome being some of the most studied pathologies. Regarding CVD, there is a need to further understand the function of gut organisms in terms of their direct impact on atherosclerotic disease in both stable and unstable plaques. It would also be interesting to understand if altering the microbiome affects pathophysiological atherosclerotic plaque formation. The paradigm should take into account host-microbial interactions at different levels (e.g., gene, protein and metabolite expression).

Knowing the unique microbiota in patients with CVD, could render it as a novel target for both predication and prevention of CVD. Appropriate consideration of personal human gut microbial composition is therefore a necessary part of personalized medicine. Such an innovative healthcare paradigm could utilize 'functional foods' or 'nutraceuticals', antibiotics or probiotics that are specifically designed to modify the individual gut microbiota and thereby change the pro-atherogenic processes in patients at risk for CAD.

\section{Financial \& competing interests disclosure}

The authors have no relevant affiliations or financial involvement with any organization or entity with a financial interest in or financial conflict with the subject matter or materials discussed in the manuscript. This includes employment, consultancies, honoraria, stock ownership or options, expert testimony, grants or patents received or pending, or royalties.

No writing assistance was utilized in the production of this manuscript.

\section{References}

1 Mozaffarian D, Benjamin EJ, Go AS et al.

Heart disease and stroke statistics - 2016 update: a report from the American Heart Association. Circulation 133(4), E38-E360 (2016).
2 Emoto T, Yamashita T, Sasaki N et al. Analysis of gut microbiota in coronary artery disease patients: a possible link between gut 
microbiota and coronary artery disease. J. Atheroscler. Thromb. 23(8), 908-921 (2016).

3 Ley RE, Peterson DA, Gordon JI. Ecological and evolutionary forces shaping microbial diversity in the human intestine. Cell 124(4), 837-848 (2006).

4 Weinstock GM. Genomic approaches to studying the human microbiota. Nature 489(7415), 250-256 (2012).

5 Human Microbiome Project Consortium. Structure, function and diversity of the healthy human microbiome . Nature 486(7402), 207-214 (2012).

6 Burcelin R. Gut microbiota and immune crosstalk in metabolic disease. Mol. Metab. 5(9), 771-781 (2016).

7 Wang Z, Klipfell E, Bennett B et al. Gut flora metabolism of phosphatidylcholine promotes cardiovascular disease. Nature 472(7341), 57-63 (2011).

8 Turnbaugh PJ, Ley RE, Mahowald MA, Magrini V, Mardis ER, Gordon JI. An obesity-associated gut microbiome with increased capacity for energy harvest. Nature 444(7122), 1027-1031 (2006).

9 Backhed F, Manchester JK, Semenkovich CF, Gordon JI. Mechanisms underlying the resistance to diet-induced obesity in germ-free mice. Proc. Natl Acad. Sci. USA 104(3), 979-984 (2007)

10 Vrieze A, Van Nood E, Holleman F et al. Transfer of intestinal microbiota from lean donors increases insulin sensitivity in individuals with metabolic syndrome. Gastroenterology 143(4), 913-916 (2012).

11 Koren O, Spor A, Felin J et al. Human oral, gut, and plaque microbiota in patients with atherosclerosis. Proc. Natl Acad. Sci. USA 108, 4592-4598 (2011).

12 Karlsson FH, Fak F, Nookaew I et al. Symptomatic atherosclerosis is associated with an altered gut metagenome. Nat. Commun. 3, 1245 (2012)

13 Kelly TN, Buzzano LA, Ajami NJ et al. Gut microbiome associates with lifetime cardiovascular disease risk profile among bogalusa heart study participants. Circ. Res. 119(8), 956-964 (2016).

14 Tang WH, Wang Z, Levison BS et al. Intestinal microbial metabolism of phosphatidylcholine and cardiovascular risk. N. Engl. J. Med. 368(17), 1575-1584 (2013).

15 Seldin MM, Meng Y, Qi H et al. Trimethylamine $\mathrm{N}$-oxide promotes vascular inflammation through signalling of mitogenactivated protein kinase and nuclear factor- $\kappa \mathrm{B}$. J. Am. Heart Assoc. 5(2), pii:e002767 (2016).
16 Wang Z, Robert AB, Buffa JA et al. Non-lethal inhibition of gut microbial trimethylamine production for the treatment of atherosclerosis. Cell 163(7), 1585-1595 (2015).

17 Li XS, Obeid S, Klingenberg R et al. Gut microbiota-dependent trimethylamine $\mathrm{N}$-oxide in acute coronary syndromes: a prognostic marker for incident cardiovascular events beyond traditional risk factors. Eur. Heart J. doi:10.1093/eurheartj/ehw582 (2017) (Epub ahead of print).

18 Tang WH, Wang Z, Fan Y et al. Prognostic value of elevated levels of intestinal microbegenerated metabolite trimethylamine- $\mathrm{N}$-oxide in patients with heart failure: refining the gut hypothesis. J. Am. Coll. Cardiol. 64(18), 1908-1914 (2014).

19 Van Nood E, Vrieze A, Nieuwdorp M et al. Duodenal infusion of donor feces for recurrent Clostridium difficile. N. Engl. J. Med. 368(5), 407-415 (2013).

20 Di Luccia B, Crescenzo R, Mazzoli A et al. Rescue of fructose-induced metabolic syndrome by antibiotics or faecal transplantation in a rat model of obesity. PLoS ONE 10(8), e0134893 (2015).

21 Karlsson FH, Tremaroli V, Nookaew I et al. Gut metagenome in European women with normal, impaired and diabetic glucose control. Nature 498(7452), 99-103 (2013). 